\title{
X-linked recessive bulbospinal neuronopathy: a report of ten cases
}

\author{
AE HARDING, ${ }^{*}$ PK THOMAS, ${ }^{*}$ M BARAITSER, $\dagger$ PG BRADBURY, ${ }^{*}$ JA MORGAN- \\ HUGHES, ${ }^{*}$ JR PONSFORD $\ddagger$
}

From the National Hospital for Nervous Diseases and Institute of Neurology, Queen Square, ${ }^{*}$ the Hospital for Sick Children, Great Ormond Street, $\dagger$ and St. Mary’s Hospital, $\ddagger$ London.

SUMMARY A form of adult onset 'bulbospinal muscular atrophy' of X-linked recessive inheritance is described in 10 patients from eight families. Muscle weakness in the limbs was mainly proximal and developed in the third to fifth decades of life, often preceded by muscle cramps on exertion and tremor of the hands. Weakness and fasciculation of the facial muscles and tongue were prominent. All the patients had gynaecomastia and some were infertile. Two had diabetes mellitus. Motor nerve conduction studies were normal but most patients had small or unrecordable sensory action potentials in the absence of clinical sensory loss. Plasma creatine kinase levels were considerably elevated and muscle biopsies showed neurogenic atrophy together with secondary myopathic changes. The importance of recognising this distinctive disorder in single cases (six of the present series) is emphasised.

The chronic spinal muscular atrophies developing in adult life are a clinically and genetically heterogeneous group of disorders. Proximal chronic spinal muscular atrophy of adult onset may be produced either by autosomal dominant or autosomal recessive genes. ${ }^{1-3}$ The same applies to the distal form of chronic spinal muscular atrophy with onset over the age of 20 years. ${ }^{45} \mathrm{~A}$ scapuloperoneal distribution of spinal muscular atrophy has been described in a small number of families in which the disorder was dominantly inherited; ${ }^{67}$ onset of symptoms is usually between the ages of $\mathbf{3 0}$ and $\mathbf{5 0}$ years. Bulbar symptoms and signs are infrequent in all these disorders.

In 1968, Kennedy and colleagues ${ }^{8}$ described a distinctive form of adult onset spinal muscular atrophy which was of $\mathrm{X}$-linked recessive inheritance. They reported two families in which 11 males initially developed proximal muscle weakness in the fourth and fifth decades of life. Muscle cramps often preceded weakness by a number of years. Facial weakness was prominent and accompanied by fasciculation. Most affected individuals had atrophy of the tongue associated with dysarthria and dysphagia. Tremor of essential type was present in some cases. Weakness, wasting and fasciculation in

Address for reprint requests: Dr AE Harding, the National Hospital for Nervous Diseases, Queen Square, London WC1N 3BG, UK.

Received 25 June 1982

Accepted 9 August 1982 the limbs spread to involve the distal musculature as the disease progressed. The tendon reflexes were usually absent. Sensation was normal. Electromyography and muscle histology confirmed the clinical impression of neurogenic atrophy, and necropsy in one case showed diffuse loss and atrophy of anterior horn cells in the spinal cord. Three of the patients had gynaecomastia and two were diabetic. Both pedigrees were compatible with $\mathrm{X}$-linked recessive inheritance. Male to male transmission occurred in one instance; however, this resulted from the marriage of an affected male to a maternal female cousin whose sister was an obligate carrier.

Similar families to those reported by Kennedy and colleagues $^{8}$ have been described by other authors, ${ }^{-12}$ all of which clearly exhibited X-linked recessive inheritance. The disorder has been poorly recognised in males without a positive family history although single cases are likely to occur in any $\mathrm{X}$-linked recessive disorder by the laws of chance without invoking the occurrence of new mutation. The present paper describes six males with $\mathrm{X}$-linked bulbospinal neuronopathy who have no known affected relatives, as well as pairs of brothers and male cousins. The importance of recognising this disease in sporadic cases will be emphasised. In addition some neurophysiological and histological findings will be presented which suggest that the disorder is not purely due to degeneration of brainstem and spinal motor neurons. 


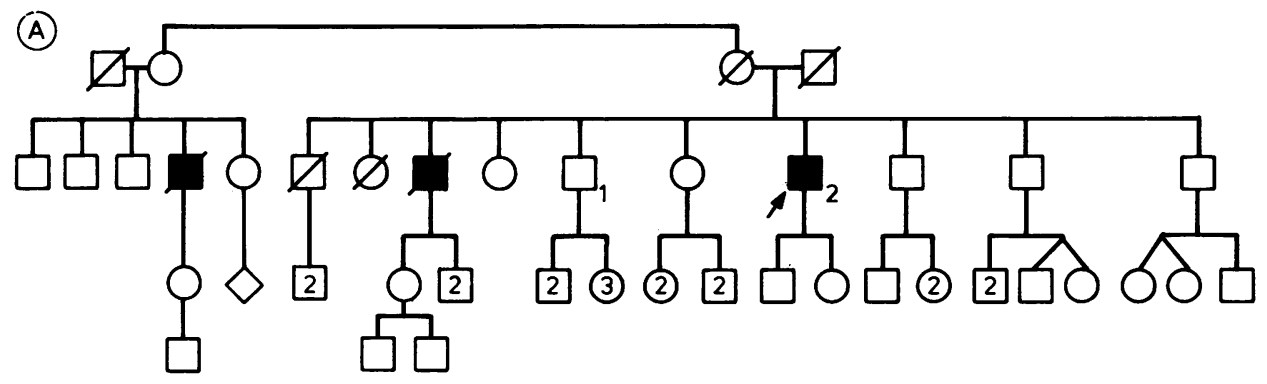

(B)

\section{Results}

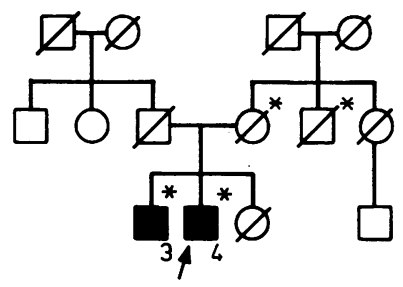

Four of the cases studied were members of two families (A and B) and their pedigrees are shown in fig 1. A third member of Family A was known to be affected but had died and no clinical details were available. Five of the remaining six patients had no similarly affected male relatives. One (Case 9) had a brother who died at the age of 52 years. He had had tremor of the hands since adolescence and
Fig 1 Pedigrees of Families $A$ and $B$ (square $=$ male, circle $=$ female, oblique line $=$ deceased, black symbols $=$ affected, open symbols $=$ unaffected, arrow $=$ index case, asterisk $=$ maturity onset diabetes).

unspecified problems related to the right leg which were attributed to cartilage dysfunction; the patient's mother had been fostered and no details were known about her relatives. Thus the pedigrees of Families A and $B$ were compatible with $X$-linked recessive inheritance and Case 9 may well have had an affected brother. The remaining five patients were single cases. The two cases from Family $B$ and a single case (Case 5) are described below for illustrative purposes. The clinical features of all 10 patients studied are summarised in table 1.

Table 1 Clinical features of 10 cases of $x$-linked bulbospinal neuronopathy

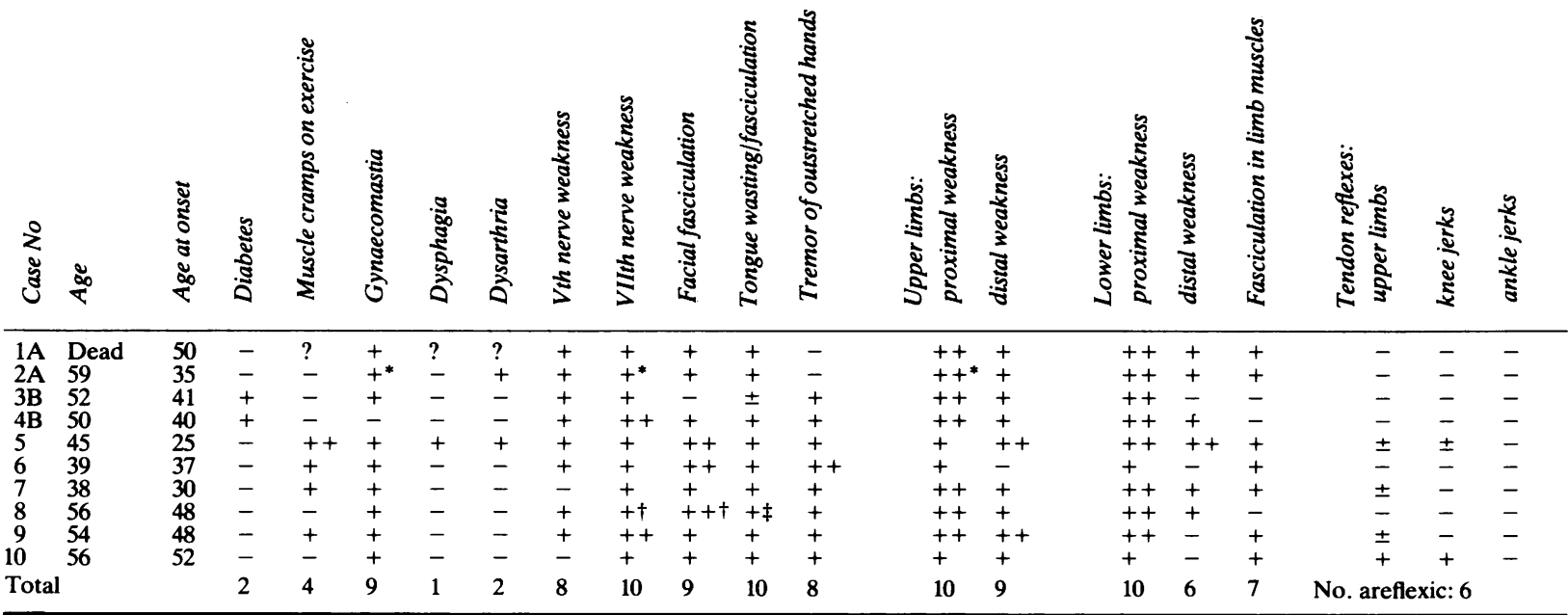

*See figure 2.

† Figure 3.

‡ Figure 4 . 


\section{Case reports}

Family B: Case 3. This 52-year-old man was found to be diabetic at the age of 40 years. The diabetes was adequately controlled with an oral glibenclamide therapy. Shortly after this he noticed progressive difficulty in climbing stairs. He also had some weakness of the right arm which was of uncertain duration. Five years prior to examination he developed acute right sided facial weakness which had since partially recovered. He had had tremor of his hands since the age of 22 years and was investigated for infertility when he was 35 .

On examination he had mild bilateral facial weakness, more marked on the right. The tongue was rather thin but not definitely wasted. There was wasting and weakness of the shoulder girdle and small hand muscles bilaterally which was more severe on the right, and bilateral proximal weakness in the lower limbs, again more severe on the right. The tendon reflexes were all absent. Vibration sense was impaired in the feet. He had bilateral gynaecomastia. There was tremor of the outstretched hands which was also present on movement.

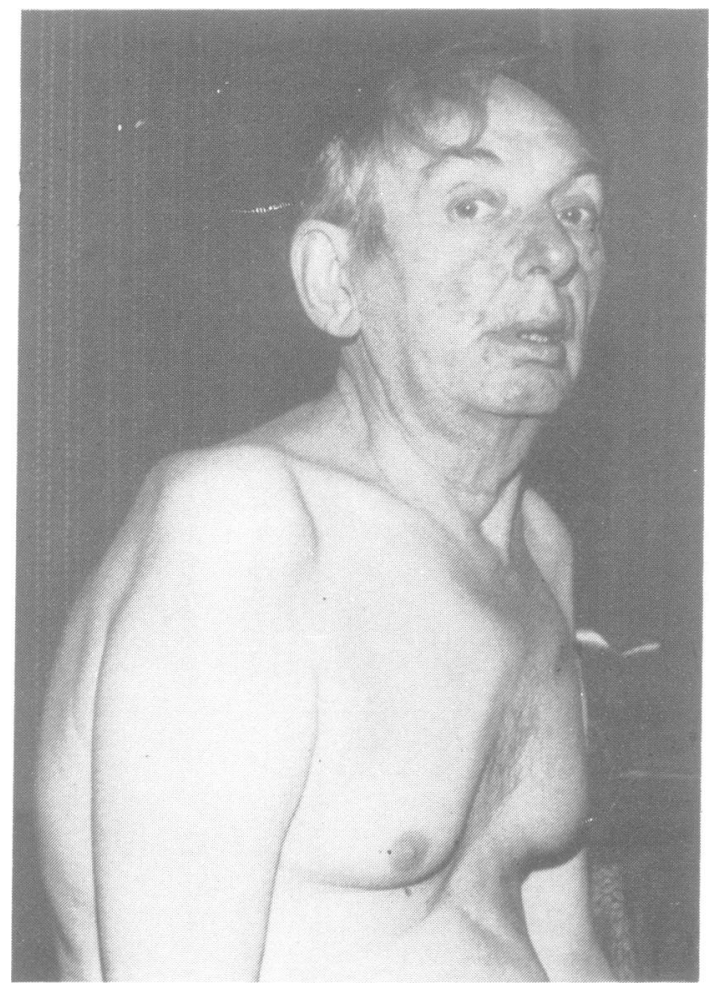

Fig 2 Case 2, showing marked wasting of the proximal upper arm and shoulder girdle muscles, and less prominent facial atrophy. There is obvious gynaecomastia.
Case 4. This .50-year-old male was investigated for infertility when he was 36 years old and was found to have azoospermia. He had been aware of progressive weakness in his arms for at least 10 years. He had had difficulty in climbing stairs for five years, and occasional tremor of his hands for many years. He had been found to have diabetes mellitus in 1972 which was subsequently treated with glibenclamide.

On examination, he had marked facial weakness and wasting and fasciculation of the tongue. There was generalised wasting and weakness of the limbs which was more evident proximally, and fasciculation in the left biceps. The tendon reflexes were all absent. The brothers' mother, who died at the age of 70 years, also suffered from maturity onset diabetes.

Case 5. This 45-year-old man developed swelling of the right breast at the age of 13 years, and of the left breast when he was 21; bilateral excision of breast tissue was performed at that time. At the age of 25 years he noticed difficulty in running and aching of the calves on exertion. When he was 30 , he became aware of wasting of his thighs, difficulty in climbing stairs and clumsiness of the hands.

On examination aged 33 he had bilateral gynae-

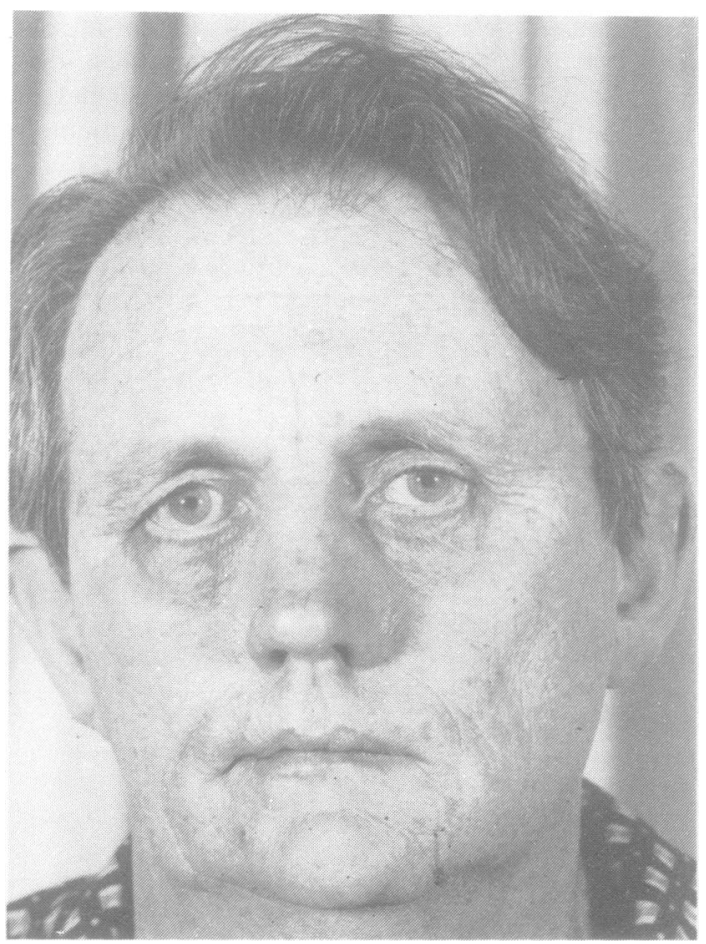

Fig 3 Case 8. There is wasting of the facial muscles; puckering around the mouth indicates the presence of fasciculation. 


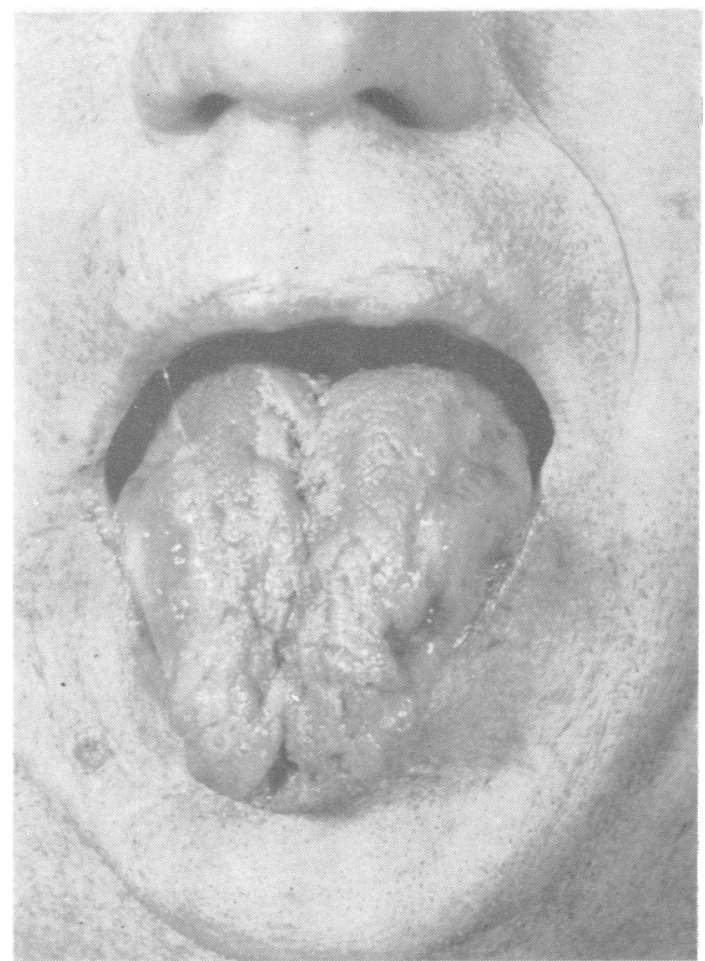

Fig 4 Wasting and fasciculation of the tongue in Case 8.

comastia. His tongue was wasted and fasciculating. In the limbs there was generalised mild wasting and weakness and prominent contraction fasciculation. Weakness was more marked distally in the upper limbs and proximally in the legs. The ankle jerks were absent and the other reflexes depressed. He was reviewed when aged 45 . His weakness had progressed and he walked with a stick. He had tended to choke on solid food for 10 years and noticed slurring of his speech occasionally. He had developed pneumonia after one particular episode of choking. On examination, there was wasting and weakness of the facial muscles with frequent spontaneous and contraction fasciculations, and tremor of the outstretched hands. Weakness, wasting and fasciculation were generalised in the limbs. The tendon reflexes were present on reinforcement except the ankle jerks which were absent. He had bilateral gynaecomastia.

\section{INVESTIGATIONS}

The results of plasma creatine kinase (CK) assays and nerve conduction studies are shown in table 2. Six of eight patients who had had CK estimations performed had elevated enzyme levels, which in one case was over five times the upper limit of the normal range. Cerebrospinal fluid examination had been performed in four cases and this was normal in all. Table 2 shows that all the patients investigated had normal or very slightly reduced motor nerve conduction velocities. However, six out of seven had small or absent sensory action potentials. If these were present, their latencies were normal. Electromyography had been undertaken in eight of the patients and showed the characteristic features of denervation (fibrillation, positive sharp waves and large polyphasic units of long duration firing at high rates with a reduced recruitment pattern). The changes were widespread, and were frequently found in clinically unaffected muscles.

Five patients had had muscle biopsies. One (Case 5) had been examined in 1966 and histochemical analysis was not performed. Routine stains showed variability in fibre size with groups of atrophic fibres and clumping of sarcolemmal nuclei. Muscle from Case 2 showed variation in fibre size with a number of small angulated fibres. There were also groups of atrophic fibres which were predominantly type I. Seven per cent showed central nuclei. There was one necrotic fibre but otherwise little structural change.

In Case 8, biopsy of the vastus medialis showed a large proportion of hypertrophied fibres with diameters ranging from 80 to $150 \mu \mathrm{m}$. Scattered throughout the section there were occasional isolated atrophic fibres, 5-10 $\mu \mathrm{m}$ in diameter, which stained intensely for oxidative enzyme activity. Some of the

Table $2 X$-linked bulbospinal neuronopathy: neurophysiological investigations and plasma creatine kinase (CK) assays

\begin{tabular}{|c|c|c|c|c|c|c|c|}
\hline \multirow[t]{2}{*}{ Case No } & \multirow{2}{*}{$\begin{array}{l}\text { Plasma } C K \\
\text { (normal range) }\end{array}$} & \multicolumn{3}{|c|}{ Sensory nerve action potential amplitude $(\mu V)$} & \multicolumn{3}{|c|}{ Motor nerve conduction velocities $(\mathrm{m} / \mathrm{s})$} \\
\hline & & Median & Ulnar & Sural & Median & Ulnar & Peroneal \\
\hline 1 & - & - & - & - & - & - & - \\
\hline 2 & $243(0-270)$ & - & - & - & - & - & - \\
\hline$\overline{3}$ & - & - & - & - & - & - & - \\
\hline 4 & $170(0-100)$ & 7 & 6 & 3 & 58 & 51 & - \\
\hline 5 & $299(10-67)$ & 21 & - & 10 & 53 & 52 & 46 \\
\hline 6 & $172(<175)$ & 3 & absent & absent & 54 & - & - \\
\hline 7 & $373(10-70)$ & & $4 \cdot 5$ & $1 \cdot 5$ & 53 & 60 & $43 \cdot 5$ \\
\hline 8 & $292(10-70)$ & absent & absent & - & - & 71 & - \\
\hline 9 & $415(10-70)$ & 2 & & 4 & 49 & 46 & - \\
\hline 10 & $581(10-70)$ & 3 & absent & absent & 46 & 57 & - \\
\hline
\end{tabular}


normal sized or hypertrophied fibres showed disorganisation of the intermyofibrillar network and loss of oxidative enzyme activity in the central regions (fig 5a). With the myofibrillar ATPase reaction, there was type 1 muscle fibre preponderance, type $2 b$ deficiency and grouping of the type 1 and type 2a fibres (fig 5b). Biopsy of the left triceps in Case 9 showed randomly scattered groups of atrophic somewhat rounded muscle fibres, scattered between hypertrophied fibres with diameters ranging from 100 to $160 \mu \mathrm{m}$. Many of the small fibres were reduced to clumps of pyknotic nuclei. The hypertrophied fibres often contained internal nuclei and some showed evidence of splitting. One or two were undergoing necrosis and phagocytosis. Some of the atrophic fibres stained intensely for oxidative enzyme activity (fig 6a). With the myofibrillar ATPase reaction at $\mathrm{pH}$ $4 \cdot 6$, there were large groups of type 1 and type $2 a$

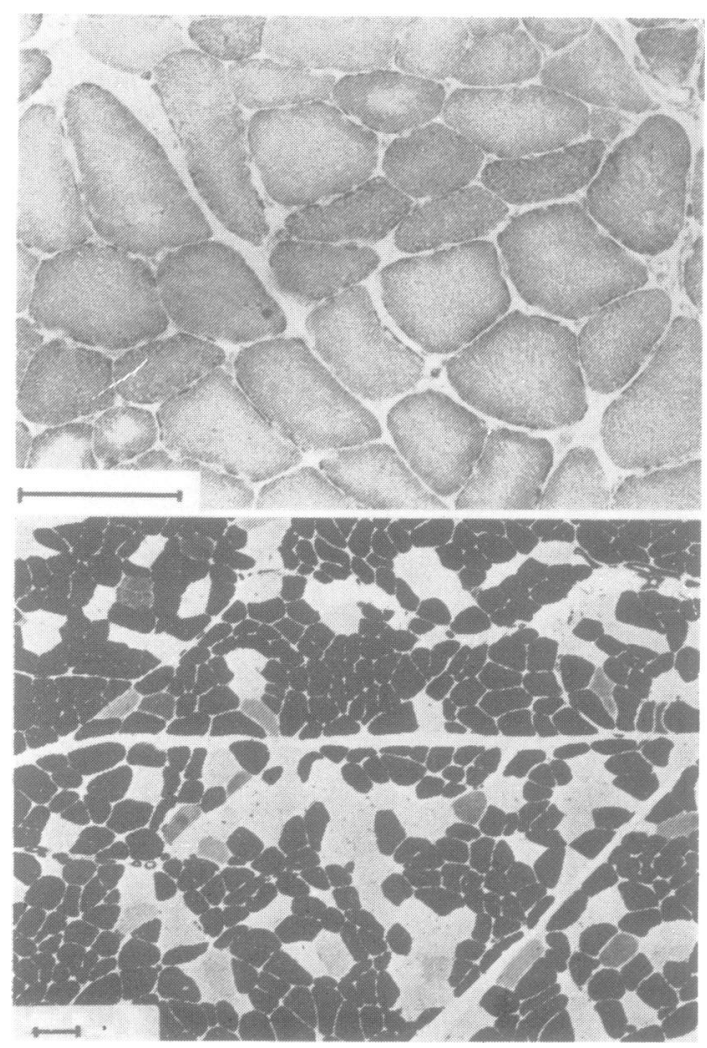

Fig 5 Biopsy of left vastus medialis from Case 8. (a), above. Hypertrophied fibres and occasional darkly staining atrophic fibres; note also the loss of oxidative enzyme activity from the central regions of some fibres; DPNH-tetrazolium reductase, bar $200 \mu \mathrm{m}$. (b), below. Myofibrillar ATPase reaction at $\dot{p} H$ 4.6 to show type 1 preponderance, type $2 b$ deficiency and grouping of the type 1 and type 2a fibres. Bar $200 \mu \mathrm{m}$.

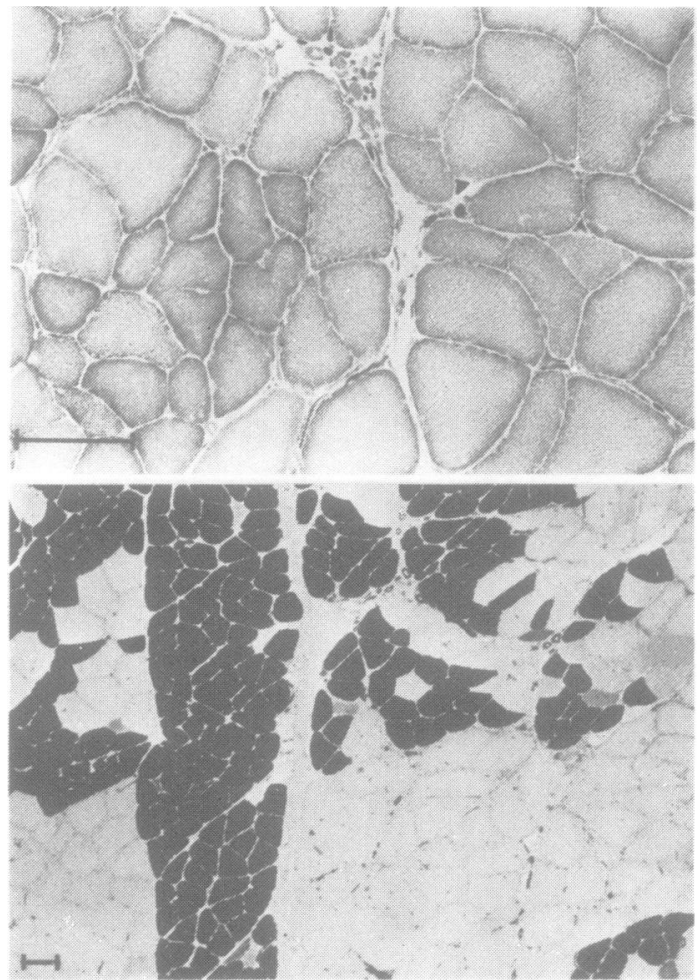

Fig 6 Biopsy of left triceps from Case 9. (a), above. A group of atrophic somewhat rounded fibres interspersed between hypertrophied fibres. Note that many of the atrophic fibres stain intensely for oxidative enzyme activity. DPNHtetrazolium reductase, bar $200 \mu \mathrm{m}$. (b), below. Myofibrillar ATPase reaction at $\mathrm{pH} 4.6$ to show type 1 and type 2 a muscle fibre grouping and a deficiency of type $2 b$ fibres. Bar $200 \mu \mathrm{m}$.

fibres with a marked deficiency of type $2 b$ fibres (fig 6b).

Biopsy of the left vastus lateralis in Case 10 showed groups of atrophic, rather rounded, fibres interspersed between markedly hypertrophied fibres. There was some splitting of the largest ones. There were frequent internal nuclei and one or two fibres were undergoing necrosis and phagocytosis. The smallest fibres stained densely for oxidative enzyme activity. With myofibrillar ATPase reactions there was evidence of early fibre type grouping.

The results of endocrine investigations, which had been performed in some of the patients, are shown in table 3. Serum prolactin was normal in all as was plasma testosterone. Two cases (3 and 7 ) had elevated plasma luteinising hormone levels, and Case 3 also had increased levels of circulating follicle stimulating hormone. Testicular atrophy was present in Cases 7 and 8, and both members of family B (Cases 3 and 4) had azoospermia. 
Table 3 X-linked bulbospinal neuronopathy: endocrine investigations

\begin{tabular}{llll}
\hline Case No & $\begin{array}{l}\text { Plasma } \\
\text { testosterone } \\
(\text { nmol/l) }\end{array}$ & $\begin{array}{l}\text { Plasma follicle } \\
\text { stimulating } \\
\text { hormone }(u / l)\end{array}$ & $\begin{array}{l}\text { Plasma } \\
\text { luteinising } \\
\text { hormone }(u / l)\end{array}$ \\
\hline 3 & $16 \cdot 1$ & $12 \cdot 0$ & $28 \cdot 0$ \\
4 & $14 \cdot 0$ & $1 \cdot 6$ & $5 \cdot 1$ \\
6 & $17 \cdot 2$ & $1 \cdot 2$ & $7 \cdot 7$ \\
7 & $22 \cdot 0$ & $5 \cdot 2$ & $9 \cdot 9$ \\
10 & $16 \cdot 0$ & $3 \cdot 9$ & $7 \cdot 4$ \\
Normal range & $9 \cdot 0-24 \cdot 0$ & $1 \cdot 0-7 \cdot 0$ & $1 \cdot 4-9 \cdot 7$ \\
\hline
\end{tabular}

\section{Discussion}

The clinical picture of bulbospinal neuronopathy is distinctive. Typically it is one of gradually progressive, mainly proximal weakness of the limbs developing in the third to fifth decades, often preceded by muscle cramps induced by exercise. Tremor of the hands may also occur before the onset of weakness. Tsukagoshi et $\mathrm{l}^{9}$ described a boy who had tremor and difficulty in writing at the age of 15 years; he did not develop limb weakness until 10 years later. One of the patients described here (Case 6) had extremely marked tremor which he found more troublesome than his mild proximal weakness 14 years after onset. The tremor was improved by propranolol. The cause of this tremor, which occurred in $80 \%$ of the present series, and a similar proportion of previously reported cases,${ }^{810}$ is unknown. It is similar to essential tremor, and that observed in chronic demyelinating neuropathies of both genetic and acquired varieties. ${ }^{13}$ It is mainly postural and is not made worse by purposive movement. The origin of the muscle cramps which preceded weakness in this series and those of other workers, ${ }^{810}$ is also unclear. They may occur in the absence of muscle weakness, as was shown by Schoenen et al $^{12}$ in a 28 -year-old male who had gynaecomastia but no other abnormal physical signs. Cramps are not uncommon in patients with motor neuron disease but rarely occur before symptoms of weakness. ${ }^{14}$

The range of age of onset in this series (25-52 years) is similar to that observed by other authors. The patient with the earliest age of onset in published reports is the boy described by Tsukagoshi et al ${ }^{9}$ referred to above. In another family, ${ }^{11}$ one male did not develop symptoms until the age of 59 years. The range of age of onset within families may vary considerably, as illustrated by Family $\mathbf{A}$ in this study and also the family of Ringel $e t a^{11}$ where the youngest patient to develop symptoms was 38 years old.

One very striking clinical feature in the present series was the presence of facial fasciculation in all but one of the patients. This was present at rest but more marked on contraction. It was most prominent around the mouth and chin and best elicited by asking the patients to whistle or blow with their mouths closed. Two of the patients were aware of involuntary facial movement. This clinical finding has been observed in over $90 \%$ of previously reported cases, ${ }^{8-12}$ and is not a common feature of any other inherited or acquired degeneration of motor nerve nuclei. Facial fasciculation in this series was accompanied by a variable degree of weakness and wasting of the muscles supplied by both the facial and the trigeminal nerves.

Fasciculation and wasting of the tongue in bulbospinal neuronopathy appears to predate the onset of bulbar symptoms. The tongue was clinically involved in all of the patients in this study. Only three were dysarthric and one had dysphagia. The latter had probably had an aspiration pneumonia. Bulbar symptoms have occurred relatively late in the course of the disease in most published cases, usually after muscle weakness has been present for at least 10 years, ${ }^{810}$ although one of the patients reported by Tsukagoshi $e t a^{9}$ had dysarthria and dysphagia at the age of 28 years. Kennedy $e^{2} a^{8}$ pointed out that, although bulbar involvement had been present for 10 to 15 years in some of their cases, it was never severe enough to interfere with nutrition. Nevertheless, one of their patients had severe bulbar symptoms and died of pneumonia. One of the cases described by Ringel et $\mathbf{l}^{11}$ had suffered from recurrent episodes of aspiration pneumonia. The life expectancy of patients with bulbospinal neuronopathy is, perhaps surprisingly, only slightly shortened if at all. The two patients who had died in this series had done so at the ages of 61 and 65 years. Kennedy $e^{2} a^{8}$ reported ages of death of $55,68,69,72$, and 83 years. The disorder is very slowly progressive; only one of our patients had severe difficulty in walking and had had to retire from work at the age of 59 years. All except one of the others were still working.

Muscle weakness and wasting were generalised in the limbs but more marked proximally in nearly all of the patients in this study. Distal involvement was more prominent in the arms than the legs; all but one had wasting of the small hand muscles, whereas in four the muscles below the knees were normal. In one case (Case 5), the distribution of weakness was more distal than proximal in the upper limbs. The tendon reflexes were completely absent in six of the patients described here. In the other four most tendon reflexes were absent and the rest depressed. They appear to be lost gradually as the disease progresses. In Case 5 , all were present except the ankle jerks 12 year prior to review, when he was virtually areflexic. In one of the patients described by Schoenen et al ${ }^{12}$ who had only 
muscle cramps and gynaecomastia at the age of 28 years, the tendon reflexes were normal.

Gynaecomastia occurred in nine of this series of cases, and has been noted in over $50 \%$ of previously published reports. ${ }^{8-12}$ In one patient described here, and in two others, ${ }^{812}$ breast excision had been performed for cosmetic reasons before the onset of neurological symptoms. Gynaecomastia would appear to be one of the manifestations of a more widespread endocrine disturbance which must result from the pleiotropic effects of the mutant gene on the $\mathrm{X}$ chromosome. Two of the patients in this series, and one of those reported by Schoenen et al ${ }^{12}$ had been investigated for infertility. Only half of our patients had reproduced, and the pedigrees presented by Stefanis $e t a^{10}$ and Schoenen et al ${ }^{12}$ suggest that biological fitness may be reduced in this disorder by a greater degree than can be explained by the extent of the patients' neurological disability. Although mild testicular atrophy was noted in one patient by Ringel et al $^{11}$ it was only observed in two patients in this study and not in any other reports. All of our cases had plasma testosterone levels in the normal range. Two had slightly elevated circulating LH and serum LH in the rest was in the high part of the normal range. This would suggest mild primary testicular failure. Schoenen et $a l^{12}$ undertook extensive endocrine investigations in two of their patients. Plasma testosterone was subnormal, and 17 B-oestradiol elevated, in both. The latter would explain the gynaecomastia. Plasma testosterone rose on administration of human chorionic gonadotrophin and dexamethasone which would suggest a primary abnormality of the hypothalamic-pituitary axis, in contrast to the findings in the patients reported here. Further dynamic tests of pituitary function are required to elucidate the endocrine manifestations of this disorder.

Another endocrine abnormality found in two patients in the current study, and some of those described by Kennedy et $a l^{8}$ was the presence of diabetes mellitus. This was of maturity onset. Two unaffected males; both sons of carrier females, were also diabetic in the family reported by Kennedy et al. ${ }^{8}$ It is of interest that the mother of Cases 3 and 4 in this series was also diabetic.

One of the patients in this series had mild loss of vibration sense in the feet but this could have been secondary to his diabetes. Another (who was not diabetic) experienced a sensation of unsteadiness if he closed his eyes but had no clinical sensory loss or Rombergism. However, he had absent or small sensory nerve action potentials, as did six out of the seven patients investigated. Abnormal sensory action potentials were also found in three of the cases by Kennedy et al $^{8}$ (two of whom were diabetic) and three out of five investigated by Stefanis et al. ${ }^{10}$ It therefore seems unreasonable to call this disorder a spinal muscular atrophy, hence our use of the term "bulbospinal neuronopathy". The site of degeneration of the sensory pathway is not known. Sural nerve histology has not been reported. Kennedy $\mathrm{et}^{\mathrm{a}} \mathrm{l}^{8}$ found a mild loss of nerve fibres in the peripheral nerves at autopsy in one case; the dorsal roots and horns were normal. However, there was slight pallor of the fasciculus gracilis in the thoracic part of the spinal cord on myelin staining.

The electromyographic abnormalities found in this study were typical of denervation, confirming the clinical impression of neurogenic atrophy. It is of interest that in most of the muscle biopsies, particularly that from Case 8 , there were a number of changes suggestive of primary muscle disease as well as those confirming denervation. The former included hypertrophied fibres, disorganisation of the intermyofibrillar network and loss of oxidative enzyme activity in the central regions of some fibres. In addition, occasional fibres in the biopsies were undergoing necrosis and phagocytosis. Prominent "secondary" myopathic changes in muscle from patients with X-linked bulbospinal spinal muscular atrophy have been described by some authors. ${ }^{101215}$ It is impossible to state with certainty whether these myopathic changes are truly secondary or not. Certainly the plasma CK levels observed in many of the patients described here, and those reported in the past, ${ }^{111215}$ were much higher than usually occur in anterior horn cell disease.

Only nine families containing individuals affected with $\mathrm{X}$-linked recessive bulbospinal neuronopathy have been reported to date which would suggest that the disorder is rare. Yet the cases described here were seen by the authors over the course of two years, which might indicate that the disease is relatively poorly recognised. It is probable that the reports of Molleen, ${ }^{16}$ Kurland, ${ }^{17}$ Magee, ${ }^{18}$ and Tsukagoshi $e t$ $a l^{15}$ describe patients with the same disease. These were labelled by the authors as having progressive muscular atrophy, amyotrophic lateral sclerosis, bulbar-spinal muscular atrophy and proximal neurogenic atrophy. Molleen, ${ }^{16}$ Magee $^{18}$ and Tsukagoshi et al $^{15}$ investigated affected brothers; the patients of Kurland ${ }^{17}$ and others of Tsukagoshi et $\mathrm{ll}^{15}$ had positive family histories suggestive of $\mathrm{X}$-linked inheritance. Although two of Magee's ${ }^{18}$ three brothers had predominantly distal weakness in the limbs, facial fasciculation was recorded which would be very suggestive of bulbospinal neuronopathy. It is of interest in relation to the neurophysiological sensory abnormalities found in the present study that Magee $^{18}$ observed pallor of the fasciculus gracilis at autopsy in one of his patients. Molleen ${ }^{16}$ found a 
reduction in the number of large myelinated fibres in the peripheral nerves in a patient who could well have had the same disease.

The main reason for the lack of recognition of $\mathrm{X}$-linked bulbospinal neuronopathy is probably that affected males are less likely either to be diagnosed as having the condition or to be reported in the literature in the absence of a family history of a similar disorder. Only one sporadic case has been described as an example of the disorder to date. ${ }^{10}$ In retrospect, it is clear that some of the patients reported by Smith and Patel, ${ }^{19}$ Gross, ${ }^{20}$ and Peters et $a l^{21}$ suffered from $\mathrm{X}$-linked bulbospinal neuronopathy. The case of Smith and Patel ${ }^{19}$ had facial fasciculation and gynaecomastia. Gross's ${ }^{20}$ patient had dysphagia, dysarthria and facial fasciculation and weakness. The case reported by Peters $e t a^{21}$ had painful muscle cramps prior to the onset of weakness. All three had wasting and fasciculation of the tongue, generalised (mainly proximal) involvement of the limbs and largely depressed or absent tendon reflexes. These patients were all male; this clinical syndrome has not, so far as the authors are aware, been described in a female.

The present study would indicate that males with $\mathrm{X}$-linked bulbospinal neuronopathy are just as likely, if not more so, to have a negative family history as they are to be aware of similarly affected relatives. Even in X-linked recessive disorders which allow reproduction by affected individuals (such as the Becker form of muscular dystrophy), single cases can be expected to be encountered frequently on statistical grounds alone, without invoking the occurrence of fresh mutants. The latter are particularly likely to occur if the biological fitness of affected males is reduced, as it is in X-linked bulbospinal neuronopathy. The recognition of such patients is of great importance, as any daughters that they have will be obligate carriers of the mutant gene and thus will have a $50 \%$ chance of transmitting the disease to their male offspring. The clinical features of $\mathrm{X}$-linked recessive bulbospinal neuronopathy are so distinctive that it should be possible to make the diagnosis with confidence, even in the absence of the disease in close relatives.

We thank Professor RW Gilliatt, Drs CRA Clarke, CJ Earl, M Gross, MJG Harrison, RS Kocen and DJ Thomas for allowing us to report patients under their care.

\section{References}

${ }^{1}$ Bundey S, Lovelace RE. A clinical and genetic study of chronic proximal spinal muscular atrophy. Brain 1975;98:455-72.
2 Pearn JH. Autosomal dominant spinal muscular atrophy. A clinical and genetic study. J Neurol Sci 1978;38:26375.

${ }^{3}$ Pearn JH, Hudgson P, Walton JN. A clinical and genetic study of adult onset spinal muscular atrophy. The autosomal recessive form as a discrete disease entity. Brain 1978;101:591-606.

${ }^{4}$ McLeod JG, Prineas JW. Distal type of chronic spinal muscular atrophy-clinical, electrophysiological and pathological studies. Brain 1971;94:703-14.

5 Harding AE, Thomas PK. Hereditary distal spinal muscular atrophy. A report on 34 cases and a review of the literature. J Neurol Sci 1980;45:337-48.

- Kaeser HE. Scapuloperoneal muscular atrophy. Brain 1965;88:407-18.

${ }^{7}$ Serratrice G, Gastaut JL, Pellissier JF, Pouget J. Amyotrophies scapulopéronières chroniques de type Stark-Kaeser (à propos de 10 observations). Rev Neurol (Paris) 1976;132:823-32.

${ }^{8}$ Kennedy WR, Alter M, Sung JH. Progressive proximal spinal and bulbar muscular atrophy of late onset. A sex-linked recessive trait. Neurology (Minneap) 1968;18:671-80.

9 Tsukagoshi H, Shoji H, Furakawa T. Proximal neurogenic atrophy in adolescence and adulthood with $\mathrm{X}$-linked recessive inheritance-Kugelberg-Welander disease and its variant of late onset in one pedigree. Neurology (Minneap) 1970;20:1188-93.

${ }^{10}$ Stefanis C, Papapetropoulos T, Scarpalezos S, Lygidakis G, Panayiotopoulos CP. X-linked spinal and bulbar muscular atrophy of late onset. $J$ Neurol Sci 1975;24:493-503.

"Ringel SP, Lava NS, Treihaft MM, Lubs ML, Lubs HA. Late-onset $\mathrm{X}$-linked recessive spinal and bulbar muscular atrophy. Muscle Nerve 1978;1:297-307.

${ }^{12}$ Schoenen J, Delwaide PJ, Legros JJ, Franchimont P. Motoneuropathie héréditaire: la forme proximale de l'adult liéé au sexe (ou maladie de Kennedy). J Neurol Sci 1979;41:343-57.

${ }^{13}$ Harding AE, Thomas PK. The clinical features of hereditary motor and sensory neuropathy types I and II. Brain 1980;103:259-80.

${ }^{14}$ Muller R. Progressive motor neuron disease in adults. Acta Psychiat Neurol Scand 1952;27:137-56.

15 Tsukagoshi H, Nakanishi T, Kondo K, Tsubaki T. Hereditary proximal neurogenic muscular atrophy in adult. Arch Neurol 1965;12:597-603.

${ }^{16}$ Molleen GA. Familial progressive muscular atrophy. Arch Neurol Psychiat 1932;27:645-60.

${ }^{17}$ Kurland LT. Epidemiologic investigations of amyotrophic lateral sclerosis III A genetic interpretation of incidence and geographic distribution. Mayo Clin Proc 1957;32:449-62.

${ }^{18}$ Magee KR. Familial progressive bulbar-spinal muscular atrophy. Neurology (Minneap) 1960;10:295-305.

${ }^{19}$ Smith JB, Patel A. The Wohlfart-Kugelberg-Welander disease. Neurology (Minneap) 1965;15:469-73.

${ }^{20}$ Gross M. Proximal spinal muscular atrophy. J Neurol Neurosurg Psychiatry 1966;29:29-34.

${ }^{21}$ Peters HA, Opitz JM, Goto I, Reese HH. The benign proximal spinal progressive muscular atrophies. Acta Neurol Scand 1968;44:542-60. 